\title{
Strategic interactions: Games of the Ju|'hoan
}

\author{
Alex de Voogt ${ }^{1}$ \\ Published online: 27 June 2017 \\ (C) Psychonomic Society, Inc. 2017
}

After the seminal work of Huizinga (1938), play has been studied as a universal trait among humans. The universality of Homo Ludens, an expression introduced by Huizinga, has stimulated the discussion on why people play, specifically, if there is an evolutionary or psychological significance to play. The explanations for play, or rhetorics, as Sutton-Smith (1997) has called them, have concentrated on a series of functions that play may exhibit in human culture. Sutton-Smith (1997, pp. 9-12) considers progress, fate, power, identity, the imaginary, the self, and frivolity each as critical to the discussions. With the possible exception of frivolity, none of these concepts can be applied universally, but together they seem to cover most of the play activities described in the literature. The literature on play, as summarized by Sutton-Smith, largely excludes games of strategy, such as board or card games. John Roberts and Garry Chick became one of few to make generalizations in sociocultural anthropology that encompass strategic games specifically.

In 1959, Roberts, Arth and Bush defined and distinguished strategy games, games of chance, and physical games. In their notion of games of strategy, physical skill must be absent and a strategy must be used; chance may or may not be involved. In their comparative research, they concluded that strategy games were not universally found among humans, a claim later confirmed by Chick (1998). Their most far-reaching claim maintained that "simple societies should not possess games of strategy and should resist borrowing them" (Roberts, Arth, \& Bush, 1959, p. 600). They considered that games are expressive, since "there are few obvious environmental and technological limitations on them" (Roberts et al., 1959, p. 598). They do not directly satisfy the biological needs associated with survival. Instead the relationships between games and needs of any single society must be complex and integrated in many different ways. They identified two general characteristics of all games: the expressive and the model 
characteristics. Specifically, they found that most games are models of various cultural activities, a statement also found in historical literature on board games, as exemplified by Murray (1952). Since they considered games expressive models, they hypothesized that "games of strategy which are models of social interaction should be related to the complexity of the social system" (Roberts et al., 1959, p. 599). The social systems were then classified by looking at low or high political integration and the absence or presence of social classes. Those with no social classes and low political integration are then thought to have fewer, if any, strategy games.

In contrast, the following analysis of the Ju|'hoan games shows that the presence of strategy games is not dependent on the complexity of a society. Similar criticism was detailed in earlier publications (Townshend, 1980; de Voogt, in press) by examining the presence of strategy games in pastoral and hunter-gatherer societies, respectively. Indeed, theories on the history and distribution of board games demonstrate that such games are specifically suitable to facilitate social interaction across cultural boundaries (Crist, de Voogt, DunnVaturi, 2016; de Voogt, Dunn-Vaturi, Eerkens, 2013). The abstract nature of the board and rules require little culturally specific knowledge so that people from different societies encounter a relatively low threshold for participating. However, there is also evidence that the Ju|'hoan, a hunter-gatherer community, developed their own strategy game, one that was not introduced by other "more complex" societies. This game, together with the other introduced strategy games, suggest that the role of games in society is better understood if contact between groups of people is taken into consideration to explain their presence rather than the rhetorics that maintain an evolutionary or psychological significance.

\section{The Ju|'hoan at |Xae|xae}

The Jul'hoan are a people living in Namibia and Botswana, and who were extensively studied in the field of anthropology during the 1960s and 1970s (Lee, 1979; Lee \& DeVore, 1976; Marshall, 1976). At that time, hunting and gathering subsistence was seen as the earliest mode of living, providing insight into how early humans might have lived in Africa. Even though this perspective is largely abandoned in anthropology, inferences to ancestral peoples made by evolutionary psychologists and biologists on contemporary practices of huntergatherers still abound (e.g., Lindsey, Brown, Brainard, \& Apicella, 2015). Within the study of play and games, theories have been put forward in which complex societies are more likely to play strategic games (Chick, 1998; Roberts et al., 1959), and where games are seen as mimicking current practices of the people who play them (Murray, 1952).

The paucity of descriptions of adult games in the anthropological literature, despite the extensive attention to the
Kalahari hunter-gatherer communities since the 1960s, is partly explained by the rubric in which games have been studied in anthropology. Lee (1979), Lee and DeVore (1976), and Schapera (1930), for instance, only mention games in the context of Ju|'hoan children. It is mostly Marshall (1976) who details games that involve both adults and children. The main exception to this practice is the seminal work by Sbrzesny (1976), who created a full account of all forms of games for the !Ko people in the Central Kalahari. This German-language work is not recognized in the mainly English-language literature on the Jul'hoan or in theoretical works about play among the San. Much of the work presented here on the games of the Ju|'hoan confirms the general findings of Sbrzesny and points to innovations and variations in the case of the Jul'hoan.

\section{Strategic games of the Ju|'hoan}

In a previous study (de Voogt, 2016), it was found that Ju|'hoan play card games, also known as Xànúsì. One of these is a version of Crazy Eights that is popular in the region and that has been acquired by children in school where exchanges with children from other culture groups take place. This game is now known and played by most adults while more complex card games, such as Casino, are only played by a few (see Fig. 1). The object of Crazy Eights is to discard one's cards as quickly as possible, which is accomplished through a series of strategic decisions. Played with two or more people, a number of cards are given special powers; for instance, the 8 allows a player to change the suit, the Jack changes the direction of play, and when the 7 is played, it skips the next player. In short, the involvement of strategy within each participant's choice of the next card is essential to winning.

The board game of this group of Ju|'hoan is a mancala game called $\mid u ́ i$, meaning "one," and which is similar to those found elsewhere in the region. The Yeyi-speaking people call it |wine and the Nama-speaking group in Namibia know the game as |lhus (Townshend, 1977; de Voogt, 2001), while in large parts of Namibia, the name is referred to as Owela. Sbrzesny (1976, pp. 156-163) found the same game with the !Ko, where the name was recorded as $|u i|$ ama !ona, meaning "one, two, three." This distribution suggests that this mancala game easily crossed cultural and linguistic borders in the southwestern part of Africa.

When playing this board game, each position allows multiple moves to be played. Ideally, complex moves are calculated for their effect on the position before they are chosen. Jul'hoan players calculate and evaluate moves consistently and play to win. The larger the size of the board (see Fig. 2), the more strategy is required to finish the game. All playing counters remain on the board during the game, which means that the game does not simplify in terms of the number of 


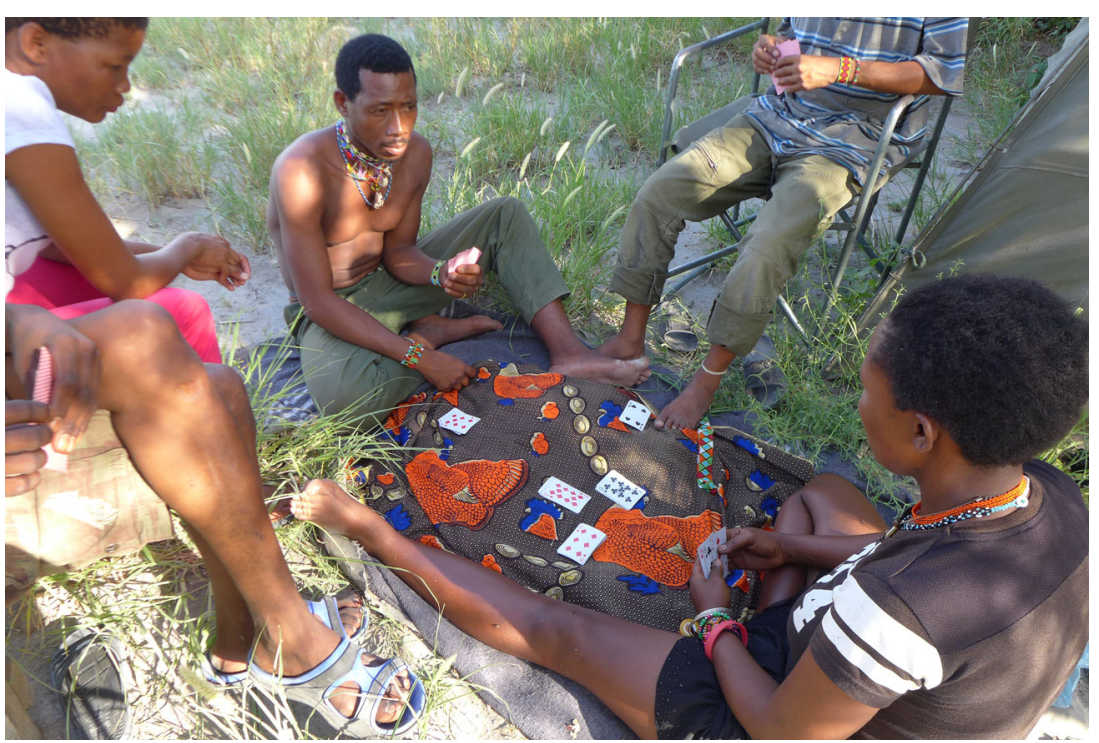

Fig. 1 Four Ju|'hoan playing the card game Casino. Photo Alex de Voogt, 2016

counters present. If one player or team is better than the other, they simply have most of the counters on their side and more moves from which to choose.

Most card and board games in the world were introduced through contact with neighboring peoples. This is amply illustrated with examples from antiquity (Crist et al., 2016; de Voogt et al., 2013). Most board and card games are not necessarily culturally specific since only few adaptations of rules and board configurations take place, an observation also found with Roberts et al. (1959) as part of their idea of the expressive nature of games in general. At most, the decorations of boards and cards vary per group. Any reasons for play, as a result, should transcend cultural boundaries to allow this contact to happen and, once the game is adopted by a neighboring society, it can take on a variety of roles.
In terms of rhetorics, the above card and board games support different discourses. Card games resonate with the idea of fate as it has an element of chance, even though it still fits the definition of strategy game formulated by Roberts et al. (1959). Board games could be part of a discussion about power and progress. However, it is noted that both women and men play these games, often with each other (see Figs. 1 and 2). At the same time, not every adult engages in these games; they are not necessary or mandatory. Consequently, any significance of play is problematic to generalize if it cannot explain why certain women and men do not participate. In an egalitarian society, such as that of the Ju|'hoan, the rhetorics on power and progress seem especially problematic, hence the prediction by Roberts et al. (1959) that these games would not be present.

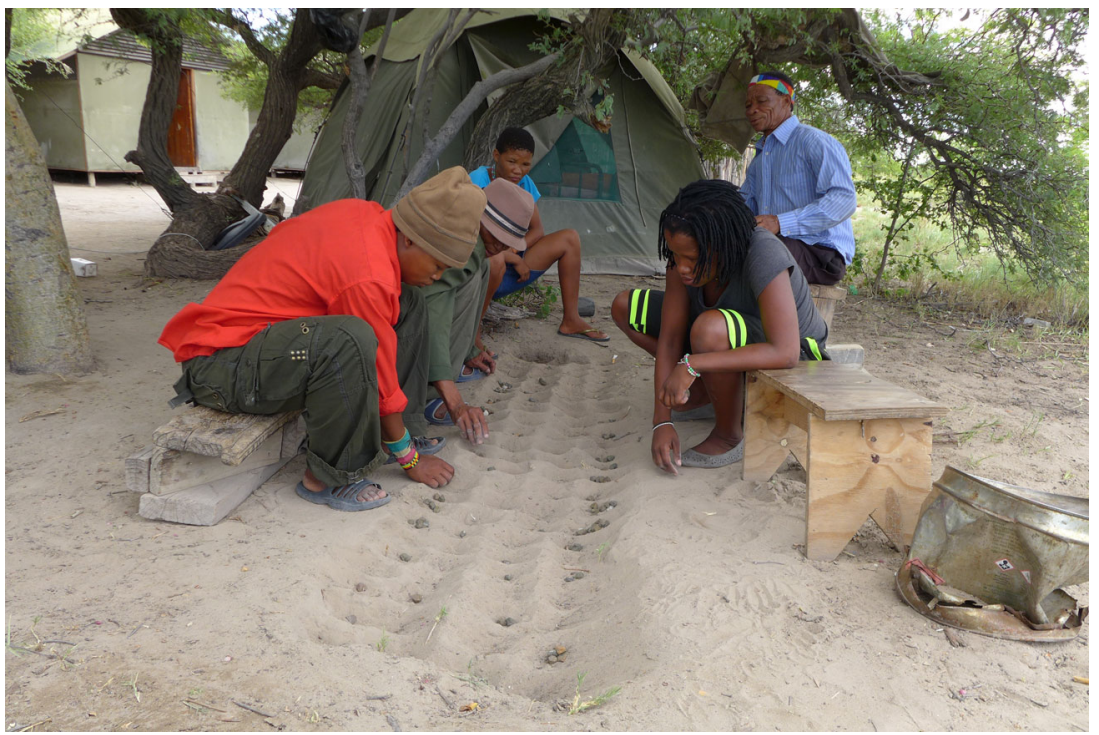

Fig. 2 Ju|'hoan playing |úí. Photo Alex de Voogt, 2016 
Finally, the Ju|'hoan play a game that involves gestures. It has been called a match-stick game or a version of rock-paperscissors, but for reasons of convenience, it is referred to here

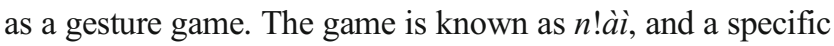
version seems to have been shared with other San communities. This game allows for rhetorics of communal identity and the self, but, as a more extensive description and analysis will demonstrate, it is also a strategy game.

\section{N!àì}

The game of $n$ !ài is regularly played and/or demonstrated by the Ju|'hoan from $|\mathrm{Xae}| \mathrm{xae}$, a village near the Namibian border on the Botswana side. A local delegation of about 16 or more people (rotating about every three months) travels about seven hours by truck to be employed at Jack's Camp, a place that offers safaris to tourists. There they organize bushwalks for guests of Jack's and two neighboring safari camps. During the walks, these guides, both men and women, commonly demonstrate the n!ài game and, alternatively, play among themselves whenever they feel like it. These games were recorded and studied for one month at Jack's Camp. Players were interviewed and also demonstrated specific aspects of the game. Insights from this study resemble previous descriptions in the literature but deviate where it comes to the interpretation and significance of the game. Elements that were seen as meaningful signifiers, such as one player representing the hunter and the other the hunted animal, are absent or have radically changed in the game of this group of players, which has "lightning" representing one side and "steenbok" the other.

One of the leading anthropologists of the Ju|'hoan, Lorna Marshall (1976, p. 332), speaks of the "porcupine," "axe and assegai," or "war" game without any distinction between them. She adds that the game is "exclusively a game for males; it is never played by women and girls, although they may stay nearby watching and joining in the laughter." She adds that Richard Lee observed "in the Dobe area, the !Kung call the game $n$ !haie ('war')," confirming her finding, but at the same time disregarding the social relevance of adult play and thus Lee's lack of any further documentation. Dickens (2009, p. 248) mentions in his dictionary of Ju|'hoan that $n$ !ài is a war-game played by men, but he mentions !'óm only as "porcupine" and not as the name of a similar game. Snyman's dictionary also speaks of a war game of men in relation to $n$ !àì. He mentions a "porcupine game" for !'óm, but without specifying that it is only played by men (Snyman, 1975, p. 120). Neither Dickens nor Snyman mention "axe and assegai" as a game - using |'ái for "axe" and tzàù̀, zaqòan or ||àqènn|ángá for “assegai.” Sbrzesny (1976, p. 143-156) found a similar game for the !Ko, but without a name. In addition, she found that the neighboring G|wi played another two variants, one of which they called gei:i, meaning "steenbok" (Sbrzesny, 1976, p. 146), a name that is given to the song accompanying this game for the Jul'hoan and that is always associated with one of the parties in the match. The other version played by the G|wi was called |aro, or "ostrich." She states that the rules do not differ in these versions, just the type of gestures in use and the accompanying sounds.

The word $n$ ! ài means "war, fight, aggression, war-game (played by men)" according to Dickens (2009). The game of n!àì, or "war," is similar to the "porcupine" game of which the players were aware and for which they pointed out some general distinctions. Their description of the porcupine game fits with the two descriptions by Marshall (1976) and Sbrzesny (1976). The distinction between these and the game of n!ài are threefold: The "porcupine" game as described by Marshall and confirmed by the Ju|'hoan from $|\mathrm{Xae}| \mathrm{xae}$ is played only in a specific context, namely during the celebrations of a successful hunt, while the "war" game can be played at any time. The "porcupine" game as well as the games played by the !Ko and G|wi are only played by men, while both men and women engage in the n!ài game. Finally, only the $n$ !ài game seems to be played in teams and includes scorekeeping.

The association of n!ài with the hunt is no longer self-evident. The two parties, commonly teams, are known as "steenbok" and "lightning," as opposed to "hunter" and some animal of choice, or "hunter" and "steenbok," as in the case of the G|wi. Despite asking elders and several different players, the game is only known using these two sides. The game is called "war," and the music that accompanies the game is called the "steenbok song," which is not used for any other game, dance, or ceremony. When asked, all these aspects have been part of the $n$ !ài game since they could remember. The "porcupine" game had always been present as a game played for a hunting ceremony, but since the state of Botswana no longer allows the Ju|'hoan to hunt, this game is no longer common.

$\mathrm{N}$ !ài is played using a style of music that is highly specific for the Jul'hoan and related groups. The rhythm of the song is essential to the operation of the game, and outsiders may grasp the basic rules of play but are particularly challenged when trying to participate in the music and identify the beats on which scoring is taking place. This culturally specific aspect of the game is essential if we wish to understand how the $\mathrm{Ju}$ |'hoan came to play this game since their use of music does not facilitate interaction with other culture groups. It was either introduced during interactions with other San groups or it was developed independently, perhaps using the "porcupine" game as a model. What is relevant is that the game is highly unlikely to have been introduced by non-San. This game and any related form of this game is not known outside the San communities, nor is it likely to have been introduced to outsiders. 


\section{Rules and strategies}

The rules of $n$ !ài can be summarized by emphasizing three elements: (1) the engagement that leads to a score, (2) the scorekeeping, and (3) the order in which the players engage in play.

1. When two players engage in play, they throw out their right or their left arm (see Fig. 3.). At a specific time determined by the music, either a left or a right arm needs to be out to realize a score. If both players have their right arm out or both have their left arm out, it is the side that identifies as "lightning" that wins. If both players have opposing arms out, right and left or left and right, it is the "steenbok" side that wins. Marshall (1976, p. 332) and Sbrzesny (1976, p. 143) mention a similarity of this game with rock-paper-scissors. This comparison is problematic as the latter also has the possibility of a draw (e.g., rockrock). In n!ài, there is always a score for one party when both players have stretched out an arm. That said, the elaborate feigning and nonsimultaneous gestures suggest that many draws of a different kind can be made before a score is established.

2. There are five wins needed to make one point, which can be interpreted as a set or a round. With each set point, the other team loses a set point. When the losing team has no points left, they remain on zero points. This rule provides a slight advantage for the team that has zero points as points are not taken away when another loss is suffered; in other words, there are no negative scores. There is no maximum or final score; players commonly quit when there is no more time or when they are getting too tired. It is noted that the chorus of nonplayers who contribute with clapping and singing is commonly the first to be exhausted.
3. If there are five people in a team, the game starts with a person sitting at one of the far ends who engages the person in front of them. The winner continues to play but engages the next in line on the opponent's team. If the opponent was to win next, this winning player would continue and engage the next player of the other team, et cetera. If all five team members of one side have lost a game, there is a set point for the opposing team. The progress of the set is thereby clearly visible, since the game moves up the line and the first to reach the far end of the line wins a set point. After a set point, the next set starts at either far end of the line; there does not seem to be a fixed rule about which side of the line is to start next.

Although the game is preferred with two teams of five, it is frequently played with fewer people, and even with unequal sizes of teams (i.e., one team may have one more member than the other team). If there are fewer than five people in a team, the same set of five is counted, but now team members may have to play again before five is reached. In a team of four people, the third person would play a second time after all four team members already played, after which the opponents could secure a point.

Sbrzesny (1976, p. 146) pointed out that both the !Ko and the $\mathrm{G} \mid$ wi have their chosen players, suggesting a difference in skill between the individual players. The Ju|'hoan at first denied that any player was better than any other. When interviewed, the players stated that once you know how to play according to the rhythm of the music, all players are equal. They did not acknowledge any particular expert or champion in the game. Although this seems to be correct with regard to the physical part of the game, there are strategies that can make a better player. The outcomes of the recorded games also confirm that the game is not determined by chance alone.

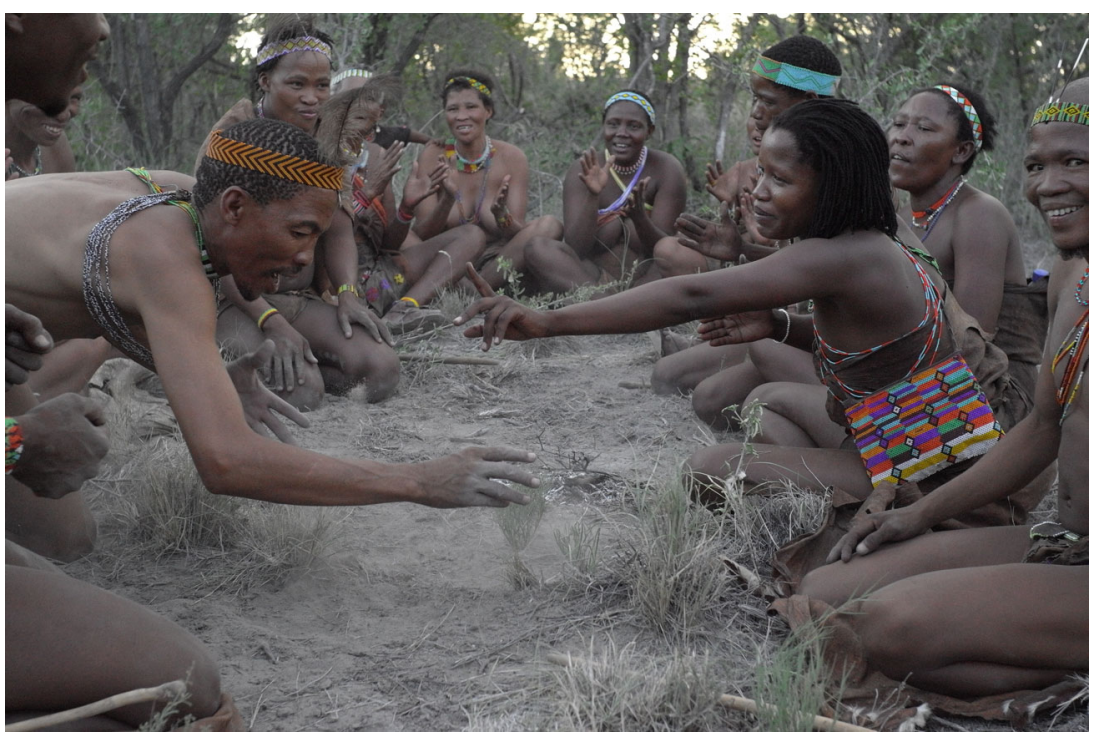

Fig. 3 Two players with outstretched arms that show the moment of a score in n!ài. Photo Alex de Voogt, 2016 
Since each set always starts at the far end of the line, those team members sitting at the far end play more games than those sitting in the middle. This information is known to the players, who position their best players at the far ends and even switch places when during the game certain players are not performing as well. It is also just the players at the far ends that can win against five players in a row; all others will have any winning streak interrupted earlier.

The beats of the music determine at which time an arm is thrown forward. Feigns are common and can be particularly elaborate. For instance, participants may not play either arm or both arms, in which case there is no score and they simply continue play until the next opportunity. Also, a player may hold out one arm and only with a gesture of a hand indicate which arm is being played. Variations are such that in some cases it is not clear to outsiders which play is being made. Only if a player seems to decide after a play which of her or his arms was in play will there be protests from the opponent commonly resolved by replaying that particular engagement.

When a machine was to play this game, the optimal strategy to win $n$ !ài is to play a random arm. People cannot play random moves consistently. Instead, their best strategy is to obfuscate their own and guess their opponent's moves. It has been shown that rock-paper-scissors, a similar game but with a possibility for a draw, has players who can beat their opponents consistently if there are a sufficient number of plays being made (Alfaro, Han, \& Schilling, 2009; McCannon, 2007).

In one long game, in which players continued for more than 20 minutes, one team had scored 10 set points with the other team down to one. In this particular exchange, one of the highest scoring players won circa $70 \%$ of the matches against one specific other player, illustrating the possible individual differences.

The scoring, strategy, and game outcomes suggest that this is not a game of chance and that it is not played as a game of chance. Also, the physical skill involved is only relevant when learning the game and does not influence the decision to play one's left or right arm. It is an elaborate strategy game that, unlike the "porcupine" game, has not lost its appeal.

\section{Identity and the self}

Sbrzesny (1976) states: "The winning points are not assembled and after the match no one is coming out as better or worse player" and "there is no competitive struggle" (p. 145). She states that "it is more or less an accident" which arm is thrown out, which would suggest that the !Ko game is a game of chance. In contrast, Marshall (1976) includes strategy by stating that "chance accounts for most victories, but if a man is alert and attentive and if he watches his adversary sharply, he notices if the adversary tends to fall into a habitual pattern of throwing out one arm or the other and can quickly vanquish him" and "a good player appears to be able to catch some clues and in a split second see which arm is coming" (pp. 335-336).

In n!ài, each interaction that is won is often accompanied by a specific gesture in which the hand and arm combine into a "throw-away" sign (see Fig. 4). There are also more elaborate or expressive displays as an effect of game-playing. Some players accompany a loss with a yell, a hand covering their face or a shaking of the head, for instance (see Fig. 5; see also Sbrzesny 1976, p. 145). Repeated losses to an opponent may be larded with feigned aggression as in grabbing a stick to hit someone. When the final set is played, there are records of celebrations where the winning party waved their behinds in the faces of the losers. All of these moments happen with laughter, meaning that they are mocking gestures rather than expressions of any animosity. A frustrated player may suddenly leave the game, in which case they continue without that person who may later rejoin. These characteristics contrast with observations witnessed by Sbrzesny as well as by Marshall. These distinctive elements are either unique to the Ju|'hoan or were simply overlooked by Sbrzesny, Marshall, and later anthropologists.

When one player was asked how the arms were supposed to move in the game, she replied that every player does that in a different way. Subsequently, each of the 10 players was asked to demonstrate their way of playing. Great variety was

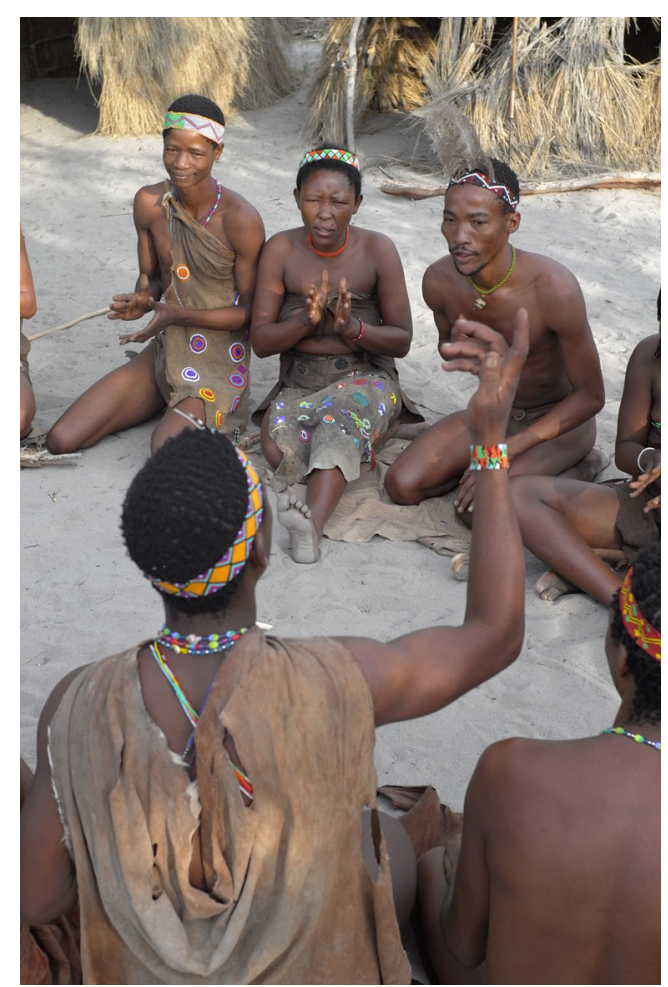

Fig. 4 Player making a "throw-away" gesture to indicate a win in the game of n!ài. Photo Alex de Voogt, 2016 


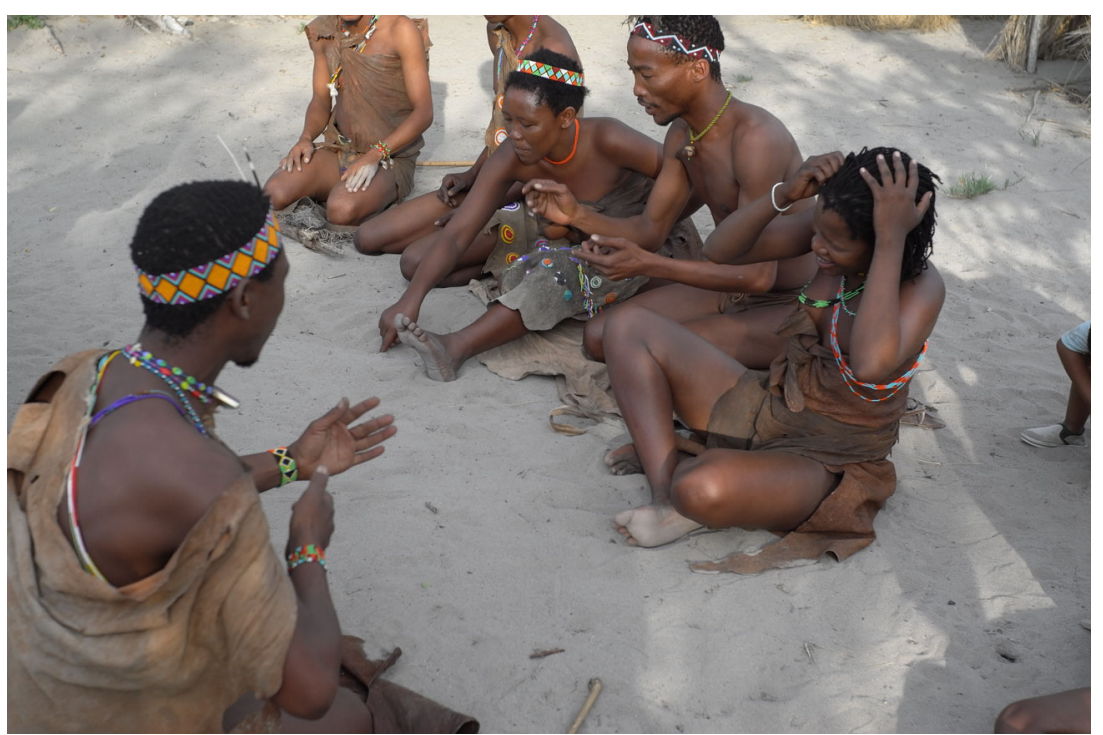

Fig. 5 One player holding her head after a loss and another player keeping score in the sand during a game of n!ài. Photo Alex de Voogt, 2016

found in the sounds that each player made to accompany their movement, the extent to which other parts of their body were included in the gesture and the positions of hands and arms in relation to the body. The players had developed an individual and expressive style, an element of Jul'hoan culture that has only recently received attention (de Voogt \& Ng, 2017). In light of this description, the game may support the rhetorics of identity and the self rather than that of power and progress as is more common for strategy games. This is also intimated by Marshall (1976), when she describes that "the other way to be a good player in !Kung estimation is to be good at pantomime. Many !Kung are highly talented in imitating human and animal movements" (p. 335). Similar suggestions in terms of identity and self are more difficult to make for Ju|'hoan card and board games for which the rhetoric of play is limited to ideas of fate, frivolity, and power. In short, each strategy game of the $\mathrm{Ju}$ |'hoan is part of a different rhetoric within the theory of play. At the same time, most if not all strategy games are primarily games for adults, who do not necessarily take part in all or any of them. It leaves the generalizations that have been made in the play literature unsatisfactory for strategy games since they cannot be made consistently, not even for a specific group of games that is played across cultures.

\section{Discussion}

The game of $n$ !ài is a strategy game. It is culturally specific since the chanting, clapping, and rhythmic scoring are part of a tradition far removed from that of neighboring peoples other than San. While the game fits ideas about cultural transmission as formulated for board games and also resonates with the idea of games as social lubricants, the rhetorics of play as well as the supposed relation between societal complexity and strategy games are challenged.

The earlier criticism on Roberts et al. (1959) and Chick (1998) is now joined by a fundamental issue, namely that an egalitarian society, such as the Ju|'hoan, has independently developed a sophisticated strategy game. Since most strategy games are introduced through neighboring societies, it is unusual to develop one's own. At most, players adapt games that they learned elsewhere, but even card games that have been introduced by European colonizers remained largely unchanged in appearance and rule set throughout the world. It makes the development of a strategy game by any group of people all the more remarkable.

Roberts et al. (1959) denied the universal presence of strategy games. This study agrees that even board and card games are not present in each community and are not played by each member of a community, either, but this is not for reasons related to the complexity of their society. Indeed, the concept of social lubricant has more explanatory power (Crist et al., 2016). The independent development of a strategy game by the $\mathrm{Ju}$ |'hoan further confirms that societal complexity is no longer a tenable explanation for the distribution of strategy games.

The game of $n$ !ài is an adult game played by both women and men in an egalitarian society. Although it is possible to support one rhetoric instead of another, depending on the game and the society in which it is played, there is no longer a general argument why adult players engage in games. It seems that the overall significance of play for children can be found in developmental aspects but that adult games constitute a separate form of play. In the view of this author, adult games are meant to engage other adults from one's own or from neighboring groups, an aspect that may already be present among children. When engaging other adults, games are competing with music, food, and drink. It makes strategy 
games particularly significant-but mostly across cultural borders.

Acknowledgements This study would not have been possible without the help of Rebecca Rivera and Mohamed Ibrahem in the video analysis of the game of $n$ !ài. I am particularly grateful for the support of Richard Butler, Elizabeth DeGaetano, Michael Turner, and the American Museum of Natural History. Finally, I wish to thank the staff and management at Jack's Camp, Botswana, especially Eugene Khumalo and Ralph Bousfield, for their generosity and kindness, but, most of all, the $\mathrm{Ju}$ |'hoan people, whose enthusiasm for this game has made this research such a pleasure.

\section{References}

Alfaro, R., Han, L., \& Schilling, K. (2009). Winning at rock-paper-scissors. The College Mathematics Journal, 40(2), 125-128.

Chick, G. (1998). Games in culture revisited: A replication and extension of Roberts, Arth, and Bush (1959). Cross-Cultural Research, 32(2), 185-206.

Crist, W., de Voogt, A. J., \& Dunn-Vaturi, A.-E. (2016). Facilitating interaction: Board games as social lubricants in the Ancient Near East. Oxford Journal of Archaeology, 35(2), 181-198.

de Voogt, A. J. (2001). Mancala: Games that count. Expedition, 43(1), $38-46$.

de Voogt, A. J. (2016). Xànúsì as played by the Ju|'hoansi in |Xae|xae, Botswana. Journal of IPCS, 44(4), 260-265.

de Voogt, A. J. (in press). Strategic games in society: The geography of adult play. International Journal of Play.

de Voogt, A. J., Dunn-Vaturi, A.-E., \& Eerkens, J. W. (2013). Cultural transmission in the Ancient Near East: Twenty squares and fiftyeight holes. Journal of Archaeological Science, 40, 1715-1730.

de Voogt, A. J., \& Ng, S. Y. (2017). Individual expression, cultural specificity and production bias in Ju|'hoan jewelry-making. Journal of Material Culture. doi:10.1177/1359183517705410
Dickens, P. J. (2009). English-Ju|'hoan, Ju|'hoan-English Dictionary. Cologne, Germany: Rüdiger Köppe Verlag.

Huizinga, J. (1938). Homo Ludens: Proeve eener bepaling van het spelelement der cultuur [Homo Ludens: Towards a definition of the element of play in culture]. Amsterdam: Tjeenk Willink.

Lee, R. B. (1979). The !Kung San: Men, women, and work in a foraging society. Cambridge: Cambridge University Press.

Lee, R. B., \& DeVore, I. (Eds.). (1976). Kalahari hunter-gatherers: Studies of the !Kung San and their neighbors. Cambridge: Harvard University Press.

Lindsey, D. T., Brown, A. M., Brainard, D. H., \& Apicella, C. L. (2015). Hunter-gatherer color naming provides new insight into the evolution of color terms. Current Biology, 25, 2441-2446.

Marshall, L. (1976). The !Kung of Nyae. Cambridge: Harvard University Press.

McCannon, B. C. (2007). Rock paper scissors. Journal of Economics, 92(1), 67-88.

Murray, H. J. R. (1952). A history of board games other than chess. Oxford: Oxford at the Clarendon Press.

Roberts, J. M., Arth, M. J., \& Bush, R. R. (1959). Games in culture. American Anthropologist, 61(4), 597-605.

Sbrzesny, H. (1976). Die Spiele der !Ko-Buschleute: unter besonderer Berücksichtigung ihrer sozialisierenden und gruppenbindenden Funktionen. MunichGermany: Piper.

Schapera, I. (1930). The Khoisan peoples of South Africa: Bushmen and Hottentots. London: Routledge.

Snyman, J. W. (1975). Zu|'hõasi fonologie \& woordeboek. Cape Town: Balkema.

Sutton-Smith, B. (1997). The ambiguity of play. Cambridge: Harvard University Press.

Townshend, P. (1977). The SWA game of $\|$ hus (das Lochspiel) in the wider context of African mankala. Journal SWA Wissenschaftliche Gesellschaft, 31, 85-98.

Townshend, P. (1980). Games of strategy: A new look at correlates and cross-cultural methods. In H. B. Schwartzmann (Ed.), Play and culture: 1978 proceedings of the Association for the Anthropological Study of Play (pp. 217-225). West Point: Leisure Press. 